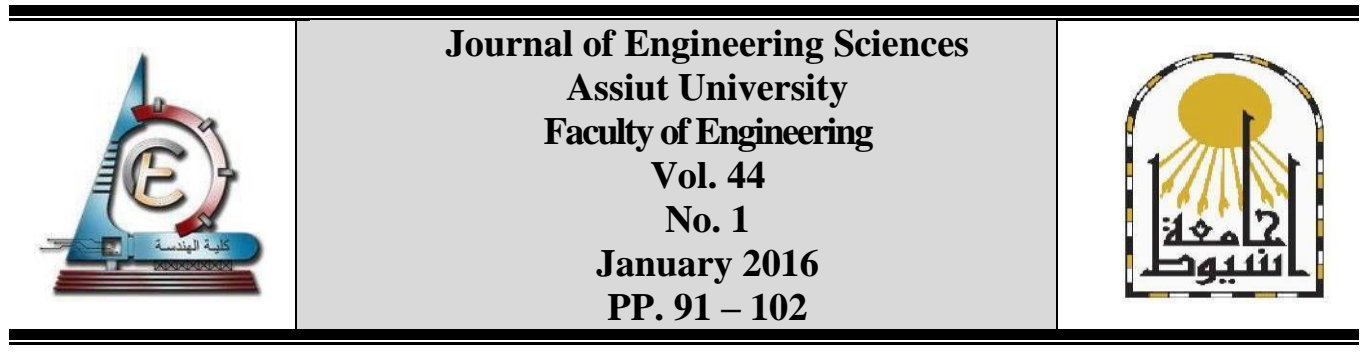

\title{
EFFECT OF CORNER MODIFICATION ON TWO- DIMENSIONAL TURBULENT FLOW AROUND A SQUARE CYLINDER WITH INCIDENCE
}

\author{
I. K. Mohamed and W. A. Aissa * \\ Mech. Power Dept., Faculty of Energy Engineering, Aswan University, Aswan, Egypt
}

Received 15 December 2015; Accepted 20 January 2016

\begin{abstract}
RNG $k-\varepsilon$ model is used to analyze the aerodynamic performance of an isolated square cylinder with different wind angles, turbulence intensities and corners shapes (sharp, chamfered and rounded). The analysis is made for unsteady two-dimensional turbulent flow. The results are presented in the form of drag coefficient, lift coefficient, RMS lift coefficient and Strouhal number. The present work is compared with previous experiments from published literature and the data gave a qualitative agreement. It is found that drag coefficient, lift coefficient, RMS lift coefficient and Strouhal number are sensitive to angle of incidence, turbulence intensity and corner modification. The average reduction in drag coefficient of square cylinder with modified corners is about $44.5 \%$ for $6.5 \%$ turbulence intensity and $41.5 \%$ for $14 \%$ turbulence intensity compared with that of square cylinder with sharp corners. Results indicated that the best fit equation of drag coefficient of sharp corners square cylinder at $45^{\circ}$ angle of incidence and $14 \%$ turbulence intensity in terms of Reynolds number; ranging from $3.0 \times 10^{4}$ to $2.825 \times 10^{5}$, is the second order polynomial equation; $C_{d}=0.0536 \times\left(\operatorname{Re} / 10^{5}\right)^{2}-0.3518 \times\left(\operatorname{Re} / 10^{5}\right)+2.0128$.
\end{abstract}

Keywords: Numerical analysis; Aerodynamic forces; Corners shape, 2D turbulent flow.

\section{Nomenclature}

$B \quad$ Breadth, (m)

$C_{d} \quad$ Time-averaged drag coefficient, (-)

$C_{L} \quad$ Time-averaged lift coefficient, (-)

$C_{l, r m s} \quad$ RMS lift coefficient, (-)

D Rectangular cylinder side, (m) and Drag, (N)

$\mu \quad$ Dynamic viscosity, $(\mathrm{kg} / \mathrm{m} . \mathrm{s})$

$v \quad$ Kinematic viscosity, $\left(\mathrm{m}^{2} / \mathrm{s}\right)$

$\alpha \quad$ Angle of incidence, (degree)

$k$ Turbulent kinetic energy $(\mathrm{J} / \mathrm{kg})$

I Turbulence intensity $=u_{1 r m s} / U$, (-)

$H \quad$ Square cylinder height, (m)

L Lift, (N)

$p \quad$ Pressure, $(\mathrm{Pa})$

$r \quad$ Corner radius, $(\mathrm{m})$

$\varepsilon \quad$ Dissipation rate of turbulent kinetic energy, (J/kg.s)

$\begin{array}{ll}\text { Abbreviations } \\ C & \text { Chamfered corners } \\ \text { LES } & \text { Large Eddy Simulation } \\ R & \text { Rounded corners }\end{array}$

* Corresponding author.

E-mail address: waessa@aswu.edu.eg 
JES, Assiut University, Faculty of Engineering, Vol. 44, No. 1, January 2016, pp. $91-102$

\begin{tabular}{|c|c|c|c|}
\hline $\operatorname{Re}$ & Reynolds number, (-) & RANS & Reynolds averaged Navier-Stocks \\
\hline St & Strouhal number, (-) & RMS & Root mean square \\
\hline $\begin{array}{ll}u_{1}, & u_{2} \\
\text { and } & u_{3}\end{array}$ & $\begin{array}{l}\text { Velocity components in } \mathrm{X}, \mathrm{Y} \text { and } \mathrm{Z} \\
\text { directions respectively, }(\mathrm{m} / \mathrm{s})\end{array}$ & RNG & Re-Normalization Group \\
\hline & Wind speed, (m) & $S$ & Sharp corners \\
\hline \multicolumn{2}{|c|}{ Greek Symbols } & $2 \mathrm{D}$ & Two dimensional \\
\hline$\rho$ & Density, $\left(\mathrm{kg} / \mathrm{m}^{3}\right)$ & $3 \mathrm{D}$ & Three dimensional \\
\hline
\end{tabular}

\section{Introduction}

Square and rectangular cylinders are major forms of bluff bodies. Various numerical and experimental studies [1-17] were performed to investigate laminar and turbulent flows past square and rectangular cylinders with different corners' shapes at zero or non-zero angle to the incoming flow. Lee [1] experimentally investigated the mean and fluctuating pressure field acting on a two-dimensional square cylinder in both cases of uniform and turbulent flows. He stated that base pressure increases, body drag decreases and vortex shedding strength decreases with turbulence intensity increase.

Tamura and Miyagi [2] analyzed experimentally the effects of corner shape and turbulence intensity on aerodynamic forces of a square cylinder in $2 \mathrm{D}$ and $3 \mathrm{D}$ turbulent flow. They concluded that corners chamfering and rounding lead to drag reduction. Further, they stated that even at zero angle of incidence, the windward edge separated flow reattaches to cylinder surface for a round-cornered cylinder.

Dutta et al. [3] experimentally investigated the flow over a square cylinder with 0 to 60 degrees incidence at Reynolds numbers varying from 97 to 187. Velocity was measured using two-component hot wire anemometer. The wake of the cylinder was visualized using a pulsed laser sheet. The effects of Reynolds number and incidence on drag coefficient, time average and RMS velocity distributions, decay of velocity fluctuations, Strouhal number and power spectra were investigated.

Letchford and Mason [4] conducted experimental investigation on 2 full-size square sections with sharp and rounded corners in 2 different turbulent flows. Results were compared with those of previous investigators.

Steggel [5] analyzed viscous and uniform (or pulsating) flow past rectangles for different values of body length-to-cross-stream dimension ratio and Reynolds number. Darekar and Sherwin [6] numerically investigated the flow over square-section cylinders having stagnation face with a sinusoidal waviness due to spanwise geometric deformation.

Ochoa and Fueyo [7] utilized Large Eddy Simulation technique to analyze turbulent vortex shedding from a bluff-body square cylinder. They applied the results to practical cases like bluff-body jet burners.

Filippini et al. [8] conducted LES of the flow past a square cylinder utilizing the selected flow conditions suggested by Rod et al. Flow visualization was utilized to clarify the flow pattern.

Almedia et al. [9] used a SIMPLEC finite volume code to simulate unsteady incompressible flows around rectangular cylinders. They validated their work with numerical and experimental results of previous investigators. 
Yamagishi et al. [10] utilized RNG $k-\varepsilon$ turbulent model to compute the flow past a square cylinder with corner cutoffs. Oil-film and mist were used to visualize surface flow pattern. They found that corners modification decreased the drag coefficient of square cylinder at zero angle of incidence.

Vikram et al. [11] used commercial CFD code fluent to investigate the flow over square body with and without corner modification for Reynolds number of 100 and 200. Results were presented in terms of lift coefficient, Strouhal number, and velocity and pressure distributions. They concluded that tangential velocity of sharp corners square cylinder is larger compared to those of rounded and chamfered corners square cylinder. In addition, they concluded that corners modification leads to reduction of the lift coefficient and width of the wake behind the square cylinder.

Ying et al. [12] conducted numerical simulations of unsteady flow around rectangular cylinders at Reynolds number of 21400 using 2D Reynolds averaged Navier-Stocks (RANS) models and 3D large eddy simulation (LES). They investigated the effects of grid quality and turbulence model on flow parameters.

Zhou et al. [13] conducted numerical investigation of the flow over a rectangular cylinder with side ratio $B / D=5$ at Reynolds number; $R e=22,000$ (based on the thickness of the cylinder) using Large Eddy Simulation. They concluded that oncoming velocity shear does not have remarkable effect on Strouhal number. Further, they stated that peak fluctuation frequency of the drag coefficient becomes identical to that of the lift coefficient with increase in velocity shear. Olawore and Odesola [14] used FLUENT software to compute two-dimensional unsteady flow over a rectangular cylinder for low Reynolds numbers. They used Gambit to generate the geometry and meshes. Further, they reported the effect of vortical structure and pressure distribution around the section of rectangular cylinders. Kavya et al. [15] conducted a numerical simulation of two dimensional unsteady flow over a square cylinder at an angle to the incoming flow for the Reynolds number $(R e)$ in the range 50-200. They presented the results in terms of Strouhal number (St), timeaveraged velocity and vorticity field. Gu and Lim [16] conducted numerical/experimental investigation of the flow past a series of rectangular bodies with different aspect ratios placed in a deep turbulent boundary layer. They compared numerical results with those obtained from wind-tunnel experiments. They concluded that the transverse width had an appreciable effect on the surface pressure around the bodies, while the longitudinal length has a little effect on the surface pressure. Bruno et al. [17] extended their benchmark study conducted in July 2008 on the aerodynamics of a stationary rectangular cylinder with chord-to- depth ratio equal to 5. They outlined the state of the art on the aerodynamics of 5:1 rectangular cylinders. They concluded that base pressure and drag coefficient obtained in different flow realizations are in good agreement. However, lift coefficient is greatly sensitive to set-up and modeling conditions.

In this study, the flow characteristics around a square section with sharp or chamfered or rounded corners at $0^{\circ}$ to 40 degrees incidence and different incoming flow Reynolds numbers and turbulence intensities by using numerical analysis are investigated. Results are validated with those presented in experimental investigations outlined by Lee [1], Tamura and Miyagi [2] and Letchford and Mason [4]. Polynomial equation is expressed which best fits drag coefficient of sharp square cylindrical cylinder at $45^{\circ}$ angle of 
incidence and $14 \%$ turbulence intensity in terms of Reynolds number in the range $3.0 \times 10^{4}$ $2.825 \times 10^{5}$. To the best of the authors' knowledge no such equation exists in the literature.

\section{Physical model}

Figure 1 shows the physical dimensions of the three configurations of the square cylinder under consideration in this study which are; a) sharp corners, b) chamfered corners of $B / 6$ length and c) rounded corners with radius of $B / 6$.

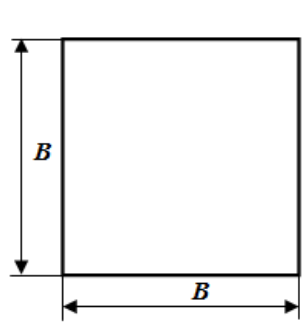

a) sharp comers
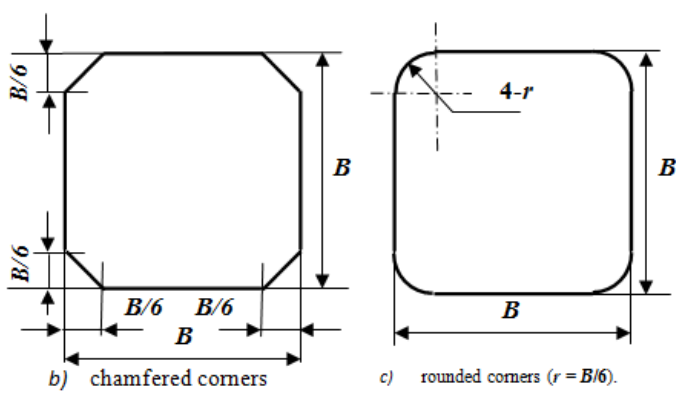

Fig. 1. physical dimensions of the three configurations under study.

Reynolds number $(\operatorname{Re}=\rho U B / \mu)$, evaluated based on body breadth $(B=50 \mathrm{~mm})$, air density $\left(\rho=1.205 \mathrm{~kg} / \mathrm{m}^{3}\right)$, air dynamic viscosity $\left(\mu=1.8207 \times 10^{-5} \mathrm{~kg} / \mathrm{m} . \mathrm{s}\right)$ and incoming wind speed $(U=9 \mathrm{~m} / \mathrm{s})$ is around $3.0 \times 10^{4}$.

\section{Numerical analysis}

RNG $k-\varepsilon$ turbulence model, mesh model, boundary conditions, and solving methodology are described as follows:

\subsection{Turbulence model}

GAMBIT grid generation code and FLUENT code are used for numerical simulations in present study. The code is used to numerically simulate the aerodynamic performance of a square cylinder using RNG $k-\varepsilon$ turbulence model with the constants of $C_{1}=1.42, C_{2}=$ 1.68 and $C_{m u}=0.0845$. Standard Navier-Stokes equation with ensemble average is used to obtain Reynolds average equation. The momentum and continuity equations are:

$$
\begin{gathered}
\frac{\partial\left\langle u_{j}\right\rangle}{\partial t}+\left\langle u_{i}\right\rangle \frac{\partial\left\langle u_{i}\right\rangle}{\partial x_{j}}=-\frac{1}{\rho} \frac{\partial\langle P\rangle}{\partial x_{i}}+\frac{\partial}{\partial x_{i}}\left[v \frac{\partial\left\langle u_{i}\right\rangle}{\partial x_{j}}-\left\langle u_{i} u_{j}\right\rangle\right] \\
\frac{\partial\left\langle u_{i}\right\rangle}{\partial x_{i}}=0
\end{gathered}
$$

Where, $\langle P\rangle$ is the average pressure, $\left\langle u_{i}\right\rangle(i=1,2,3)$ is the average velocity component $X, Y$ and $Z, \rho$ is the air density, $v$ is the air kinematic viscosity and $-\rho\left\langle u_{i} u_{j}\right\rangle$ is the Reynolds stress which lead the equation unseal.

\subsection{Flow domain}

The flow past a square cylinder with and without corner modification is considered as the main problem of this study. A calculation domain used for the current investigation is shown in figure 2. The size of the calculation domain; which is rectangular area, is $50 B \times$ $20 B$. The field extended $10 B$ upstream and $40 B$ downstream of the square section 
respectively. It should be expected that there is a great gradient change nearby the section area. A structured grid system with the grid number of $1 \times 10^{5}$ is used to resolve the flow with the first grid $\delta=0.02 \mathrm{~B}$ on the body surface.

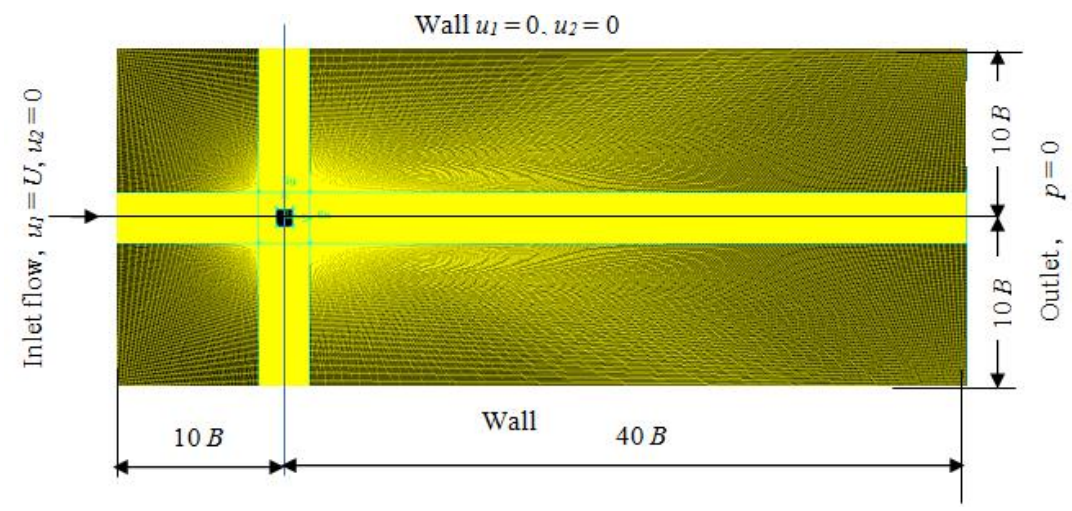

Fig. 2. Grid and boundary conditions used for computation.

\subsection{Boundary conditions}

In the present investigation, flow past square cylinder with and without corner modification has been computed by applying boundary conditions as follows.

(a) Inlet flow $\left(u_{1}=U=9 \mathrm{~m} / \mathrm{s}, u_{2}=0.0\right)$,

(b) Cylinder surface -No slip $\left(u_{1}=0.0, u_{2}=0.0\right)$,

(c) Inlet turbulence intensity; $I=6.5 \%$ and $I=14 \%$,

(d) Top and bottom boundaries-symmetry boundary condition,

(e) Outlet boundary-outflow boundary condition.

\subsection{Solving methodology}

The implicit unsteady 2D algorithm is adopted with time step of $0.001 \mathrm{~s}$. Pressure and velocity coupling SIMPLE algorithmic is used. Second order upwind scheme is used for calculating the momentum, turbulence and dissipation rate. Iteration residual less than $10^{-4}$ is the convergent condition.

\section{Results and discussion}

Drag and lift coefficients based on projected area $\left(C_{d}\right.$ and $\left.C_{L}\right)$ are defined as:

$$
C_{d}=\frac{D}{\left(\frac{1}{2} \rho U^{2}\right) B H(\operatorname{Cos} \alpha+\operatorname{Sin} \alpha)} \& C_{L}=\frac{L}{\left(\frac{1}{2} \rho U^{2}\right) B H(\operatorname{Cos} \alpha+\operatorname{Sin} \alpha)}
$$

where, $D$ and $L$ are drag and lift respectively, $\alpha$ is angle of incidence; Figure 3 , and $H$ is square cylinder height. 


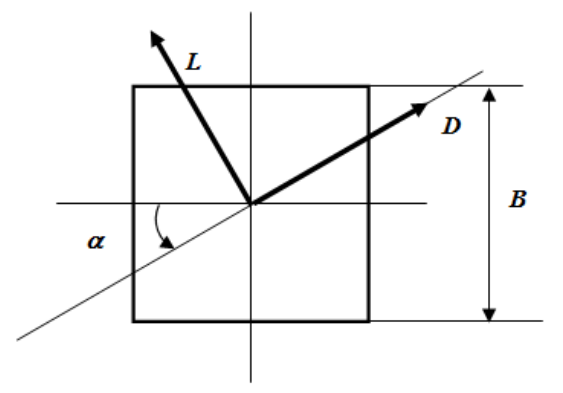

Fig. 3. Definition of the aerodynamic coefficients.

Figures 4 and 5 present the variation of drag coefficient of sharp corners square cylinder with Reynolds number for $14 \%$ turbulence intensity at $0^{\circ}$ and $45^{\circ}$ angles of incidence, respectively. It was stated in [4] that the maximum value of the drag coefficient of smooth flow past square cylinder with sharp corners at zero angle of incidence is about 2 and that the values of drag coefficients for turbulent flow are less than the corresponding smooth flow values for sharp and rounded corners square cylinders.

It may be remarked from figure 4 that the values of drag coefficient of sharp

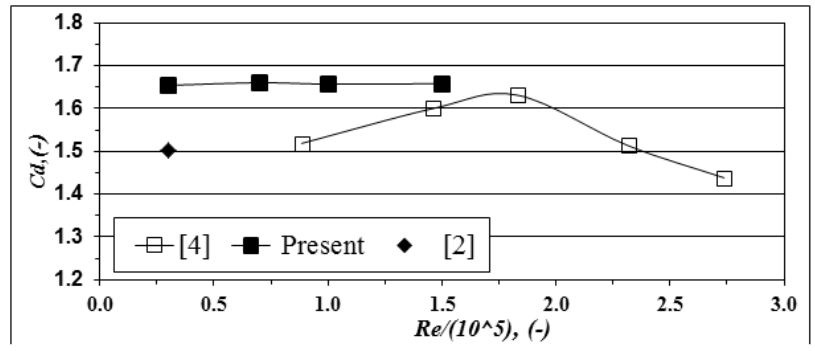

Fig. 4. Turbulent flow mean drag coefficient of sharp square as a function of Reynolds No. ( $\alpha$ $\left.=0^{\circ}, I=14 \%\right)$.)

corners square cylinder at zero angle of incidence are less than 2.0, almost independent of Reynolds number compared to those in [4].

It may be remarked from figure 5 that drag coefficient of sharp corners square cylinder at $45^{\circ}$ angle of incidence and $14 \%$ turbulence intensity decreases with increasing Reynolds number. This matches findings presented in [4]. This might be attributed as follows, with increasing Reynolds number the vortices form further from the base, resulting in decreased drag. Curve fitting of drag coefficient of sharp corners square cylinder at $45^{\circ}$ angle of incidence and $14 \%$ turbulence intensity versus Reynolds number in the range $3.0 \times 10^{4}-2.825 \times 10^{5}$; plotted in figure 5, yielded the second order polynomial equation; $C_{d}=0.0536 \times\left(\operatorname{Re} / 10^{5}\right)^{2}-0.3518 \times\left(\operatorname{Re} / 10^{5}\right)+2.0128$. 
W. A. Aissa et al., Effect of Corner Modification on Two-Dimensional Turbulent Flow Around ...

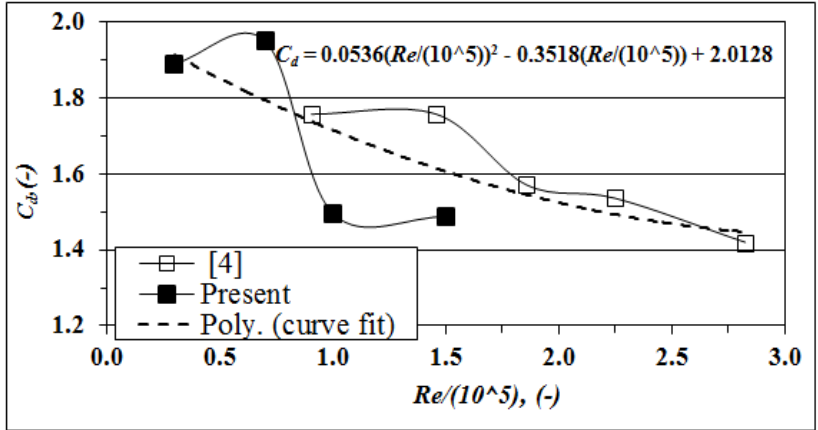

Fig. 5. Turbulent flow mean drag coefficient of sharp square cylinder as a function of Reynolds No. $\left(\alpha=45^{\circ}, I=14 \%\right)$.

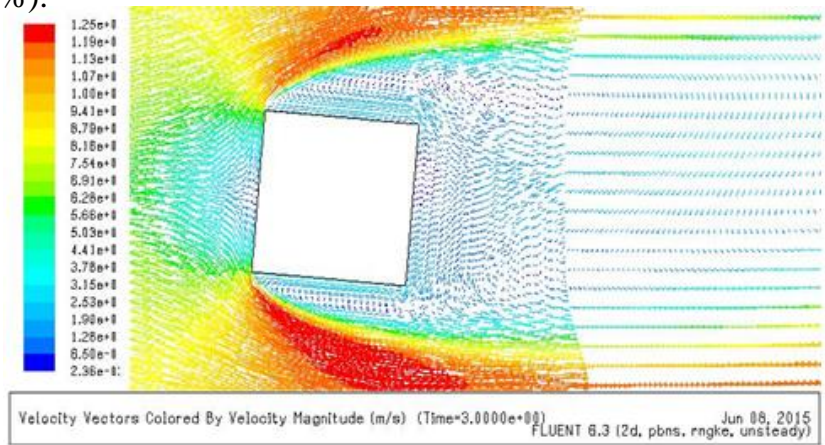

(a) sharp corners

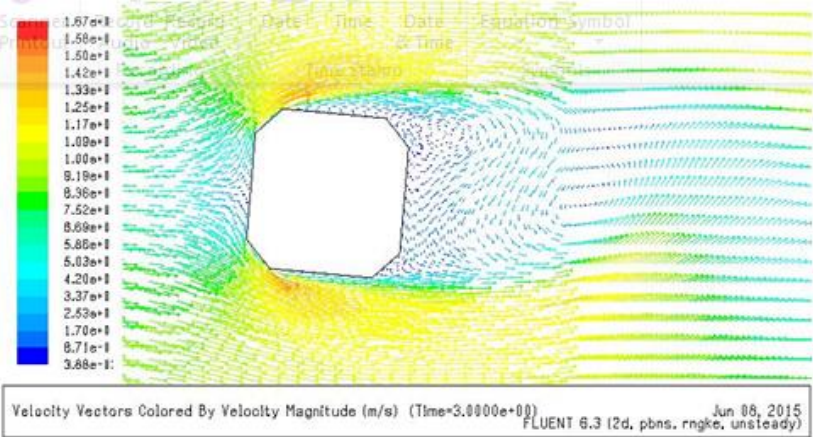

(b) chamfered corners

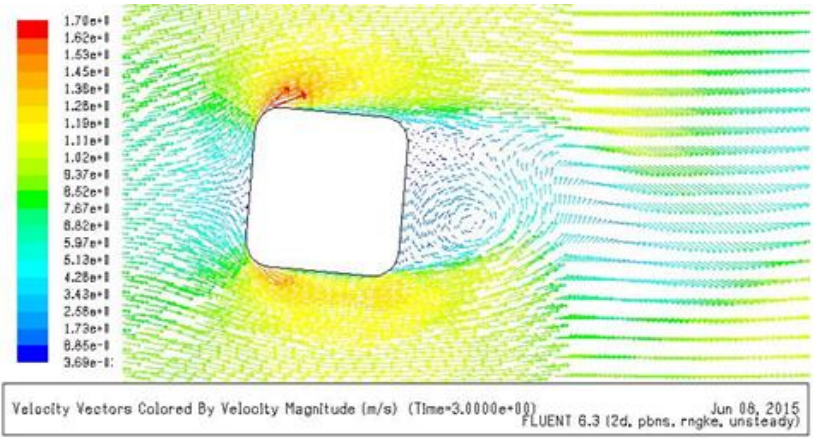

(c) rounded corners

Fig. 6. Velocity vectors around $2 \mathrm{D}$ square cylinder with different corner shapes in turbulent flow $\left(\alpha=5^{\circ}, I=6.5 \%, R e=3.0 \times 10^{4}\right)$ 
To clarify the characteristics of 2D turbulent flow around a square cylinder with corners modification; chamfering or rounding, velocity vectors, drag and lift coefficients and Strouhal number are plotted for various corner shapes. Figure 6 shows the velocity vectors around sharp, chamfered and rounded corners square cylinder. It may be remarked that flows separating from windward edge of square cylinder with modified corners begin to reattach to the cylinder surface. It may be remarked also that separation areas for side face of square cylinders with chamfered corners; Figure $6 \mathrm{~b}$, and with rounded corners; Figure $6 \mathrm{c}$, are small compared with that of sharp corners; Figure $6 \mathrm{a}$.

Figures $7 \& 8$ illustrate the variation of drag \& lift coefficients with angle of incidence for $2 \mathrm{D}$ square cylinders with different corners shapes at turbulence intensities of $6.5 \%$ and $14 \%$ and corresponding values presented in Tamura and Miyagi [2] at 14\% turbulence intensity. It may be remarked from figure 7 that drag is appreciably decreased by corner modification. This may be attributed from figure 6 that corners modification lead to reattachment of the flow on the sides of square after leading edge separation and reduction in wake width. Reattached flow leads to pressure recovery, hence less reduction in the pressure on the back face of the square cylinder. Further, it may be remarked from figure 7 that for the whole range of angle of incidence, drag coefficient of square cylinders with rounded corners is less than that of chamfered corners square cylinders which in turn is less than that of sharp corners square cylinders. This is valid for both current results and those presented by Tamura and Miyagi [2].

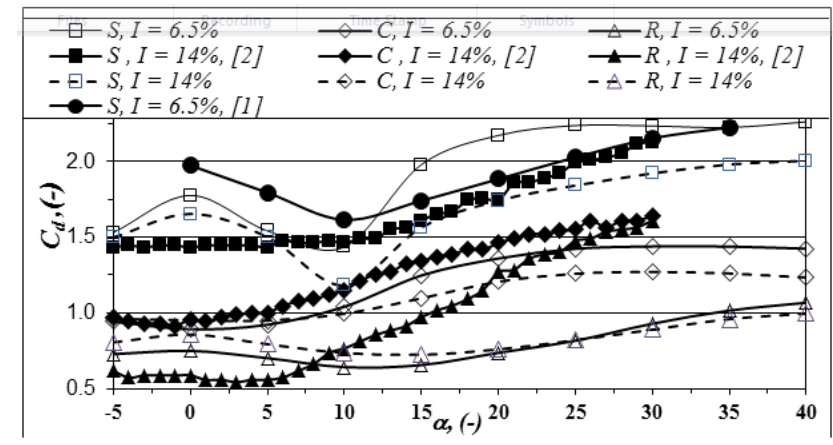

Fig. 7. Effect of corner shape on $C_{d}$ in turbulent flow for 2D square cylinders; validation with analogous results in [2], $\left(R e=3.0 \times 10^{4}\right)$.

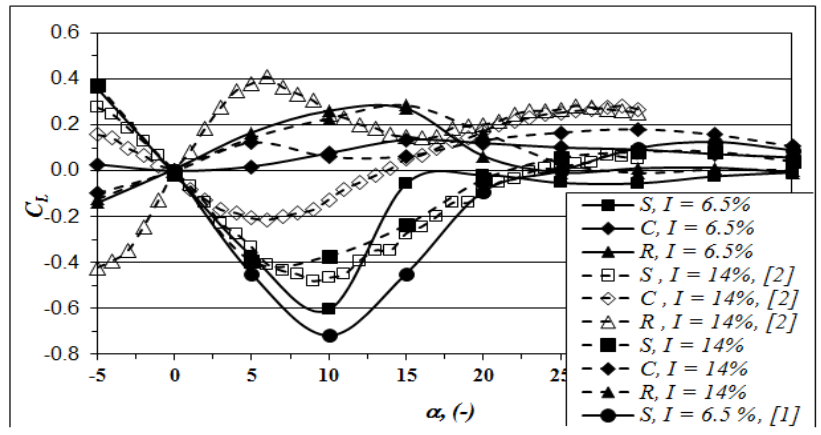

Fig. 8. Corner shape effect on $C_{L}$ in $2 \mathrm{D}$ turbulent flow for square cylinders; validation with analogous results in [2], $\left(R e=3.0 \times 10^{4}\right)$. 
The average reduction in drag coefficient of square cylinder with chamfered corners is about $37.5 \%$ for $6.5 \%$ turbulence intensity and $33.0 \%$ for $14 \%$ turbulence intensity compared to that of square cylinder with sharp corners. However, for square cylinder with rounded corners, the average reduction in drag coefficient is about $58.0 \%$ for $6.5 \%$ turbulence intensity and $50.0 \%$ for $14 \%$ turbulence intensity compared to that of square cylinder with sharp corners. In addition, it may be concluded that minimum value of the drag coefficient of square cylinder with corner modification is at an angle of incidence of about $\alpha=0 \sim 10^{\circ}$. This matches findings listed in [10].

Further, it may be observed that drag coefficient decreases with turbulence intensity increase. This matches results presented by Lee [1] and Tamura and Miyagi [2]. This might be attributed as follows; as turbulence intensity increases, base pressure increases, and vortex shedding strength decreases, hence body drag decreases.

Figure 8 illustrates a $C_{L}$ qualitative agreement of this study with that of Tamura and Miyagi [2]. The slope of $C_{L}\left(\mathrm{~d} C_{L} / \mathrm{d} \alpha\right)$ is negative for sharp and chamfered corners square cylinder while it is positive for rounded corners at an angle of incidence; $\alpha=0^{\circ}$. Thus, a complete separation flow patterns are in the case of sharp and chamfered corners square cylinders while a separation with reattachment are in the case of rounded corners square cylinder in turbulent flow.

Figure 9 illustrates the variation of RMS lift coefficient with angle of incidence for different corners shapes at turbulence intensities of $6.5 \%$ and $14 \%$ and corresponding values presented by Tamura and Miyagi [2] at 14\% turbulence

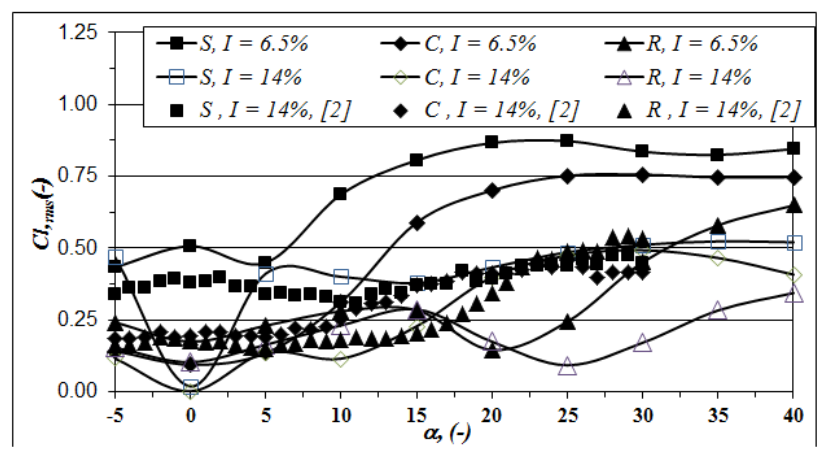

Fig. 9. Corner shape effect of on $C_{l, r m s}$ in $2 \mathrm{D}$ turbulent flow for square cylinders; validation with analogous results in [2], $\left(R e=3.0 \times 10^{4}\right)$.

intensity. It may be observed that corners modification leads to reduction in RMS lift coefficient. This matches trend of corresponding values presented by Tamura and Miyagi [2] at $14 \%$ turbulence intensity.

Vortex shedding frequency $(f)$ is related to the wind speed $(U)$ and to the projected dimension normal to the flow direction $\{B(\cos \alpha+\sin \alpha)\}$ through the Strouhal number $(S t)$, defined as [3]:

$$
S t=\frac{f B(\cos \alpha+\sin \alpha)}{U}
$$

Figure 10, illustrates variation of Strouhal number versus angle of incidence of a square cylinder with different corners shapes for turbulence intensities of $6.5 \%$ and $14 \%$ and corresponding values presented by Tamura and Miyagi [2] for 14\% turbulence intensity. It 
was stated in [2] that there is a specific angle at which the slope of $C_{L}$ function $\left(\mathrm{d} C_{L} / \mathrm{d} \alpha\right)$ changes from negative to positive. This is valid for sharp or chamfered or rounded corners square cylinder. It may be observed that Strouhal number of rounded corners square cylinder is greater than that of chamfered corners square cylinder which in turn is greater than that of sharp corners square cylinder. Further, it may be remarked that Strouhal numbers of square cylinder with chamfered and rounded corners are increased with turbulence. It was stated in [2] that Strouhal number jump for the rounded corner square cylinder at $13^{\circ}$ angle of incidence may be attributed to separation point change.

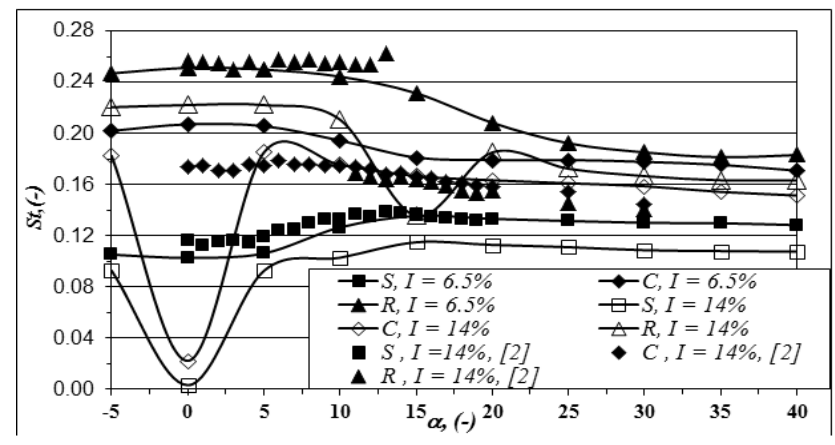

Fig. 10. Corner shape effect on $S t$ in $2 \mathrm{D}$ turbulent flow for square cylinder; validation with analogous results in [2], $\left(R e=3.0 \times 10^{4}\right)$.

\section{Conclusions}

The effect of corner modification on drag coefficient, lift coefficient, RMS lift coefficient and Strouhal number in the case of unsteady two-dimensional turbulent flow past square cylinders with incidence is investigated numerically using RNG $k-\varepsilon$ turbulence model. The results of the numerical analysis led to the following conclusions:

- The average reduction in drag coefficient of square cylinder with modified corners is about $44.5 \%$ for $6.5 \%$ turbulence intensity and $41.5 \%$ for $14 \%$ turbulence intensity compared with that of square cylinder with sharp corners.

- Best fit equation of drag coefficient of sharp corners square cylinder at $45^{\circ}$ angle of incidence and $14 \%$ turbulence intensity in terms of Reynolds number; ranging from $3.0 \times 10^{4}$ to $2.825 \times 10^{5}$, is the second order polynomial equation; $C_{d}=$ $0.0536 \times\left(\operatorname{Re} / 10^{5}\right)^{2}-0.3518 \times\left(\operatorname{Re} / 10^{5}\right)+2.0128$

- Drag coefficient of sharp corners square cylinder at zero angle of incidence and 14 $\%$ turbulence intensity is almost independent of Reynolds number.

- Drag coefficient of sharp corners square cylinder at $45^{\circ}$ angle of incidence and $14 \%$ turbulence intensity decreases with increasing Reynolds number.

- Corners rounding promote the separated shear layers reattachment.

- Drag is appreciably decreased by corner modification and turbulence intensity increase.

- Corners modification leads to reduction in RMS lift coefficient.

- Strouhal number of square cylinder with rounded corners is greater than that of chamfered corners and sharp corners square cylinders.

- Strouhal numbers increase due to turbulence for chamfered and rounded corners square cylinders.

- Present numerical model gives a fair agreement with the pervious experiments. 


\section{Acknowledgments}

The authors acknowledge with thanks the valuable remarks of the reviewers which contributed in enhancing the attitude of the paper.

\section{REFERENCES}

[1] Lee, B. E., "The effect of turbulence on the surface pressure field of a square prism," J. Fluid Mech. 1975, 69 (2), pp. 263-282.

[2] Tamura, T. and Miyagi, T., "The effect of turbulence on aerodynamic forces on a square cylinder with various corner shapes," Journal of Wind Engineering and Industrial Aerodynamics 1999; 83, pp. 135-145.

[3] Dutta, S., Panigrahi, P. K. and Muralidhar, K., "Effect of orientation on the wake of a square cylinder at low Reynolds numbers," Indian Journal of Engineering \& Materials Sciences 2004, 11, pp. 447-459.

[4] Letchford. C. and Mason, M., "Drag of square section tubes," 13th International Conference on Wind Engineering, July 10-15, 2011, Amsterdam, Netherlands.

[5] Steggel, N., "A Numerical Investigation of the Flow Around Rectangular Cylinders," PhD Thesis, University of Surrey, U.K., March 1998, p. 234.

[6] Darekar, R. M. and Sherwin, S. J., "Flow past a square-section cylinder with a wavy stagnation face," J. Fluid Mech. 2001, 426, pp. 263-295.

[7] Ochoa, J. S. and N. Fueyo, "Large Eddy Simulation of a flow past a square cylinder," International PHOENICS Conference, Melbourne, Australia, May 2004.

[8] Filippini, G., Franck, G., Nigro, N., Storti, M. and D'Elia, J. 'Large Eddy Simulations of the Flow Around a Square Cylinder,” Mecánica Computational 2005, XXIV, pp. 1279-1297.

[9] Almedia, O., Mansur, S. S. and Silveira-Neto, A. "On The Flow Past Rectangular Cylinders: Physical Aspects and Numerical Simulation," Engenharia Térmica (Thermal Engineering) 2008, 7 (1), pp. 55-64.

[10] Yamagishi, Y., Kimura, S., Oki, M. and Hatayama, C., "Effect of corner cutoffs on flow characteristics around a square cylinder," 10th International Conference on Fluid Control, Measurements, and Visualization, August 17-21, 2009, Moscow, Russia.

[11] Vikram, C.K., Krishne Gowda, Y.T. and Ravindra, .H.V., "Influence of corner cutoffs on flow past square cylinder," International Conference on Challenges and Opportunities in Mechanical Engineering, Industrial Engineering and Management Studies 698 (ICCOMIM 2012), 11-13 July, 2012.

[12] Ying, X., Xu, F. and Zhang, Z., "Numerical simulation and visualization of flow around rectangular bluff bodies," The Seventh International Colloquium on Bluff Body Aerodynamics and Applications (BBAA7) Shanghai, China; September 2-6, 2012.

[13] Zhou, Q., Cao., S. and Zhou, Z., "Shear effects on flow past a rectangular cylinder with side ratio $\mathrm{B} / \mathrm{D}=5$," The Seventh International Colloquium on Bluff Body Aerodynamics and Applications (BBAA7) Shanghai, China; September 2-6, 2012.

[14] Olawore, A.S. and Odesola, I. F. "2D Flow around a rectangular cylinder: A computational study,” AFRREV STECH Journal 2013, 2 (1), pp. 1-26.

[15] Kavya, H. P., Kotresha, B. and Naik, K. "CFD Analysis of 2-D unsteady flow past a square cylinder at an angle of incidence," International Journal of Advanced Research in Mechanical and Production Engineering and Development 2014, 1(2), pp. 117-125.

[16] Gu, D. and Lim, H. C., "Wind flow around rectangular obstacles and the effects of aspect ratio," The Seventh International Colloquium on Bluff Body Aerodynamics and Applications (BBAA7) Shanghai, China; September 2-6, 2012.

[17] Bruno, L., Salvetti, M. V. and Ricciardelli, F. "Benchmark on the Aerodynamics of a rectangular 5:1 cylinder: an overview after the first four years of activity," Journal of Wind Engineering and Industrial Aerodynamics 2014, 126, pp. 87-106. 


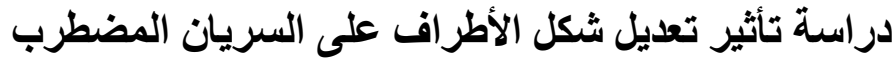

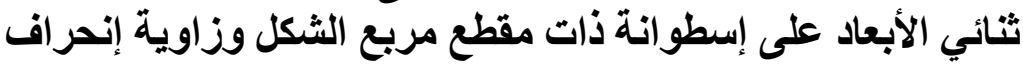

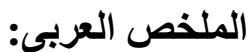

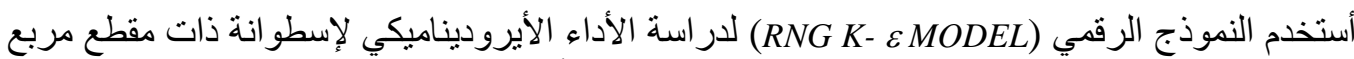

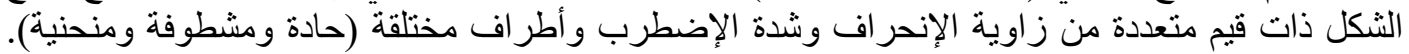

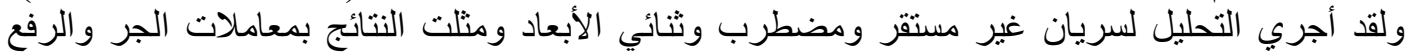

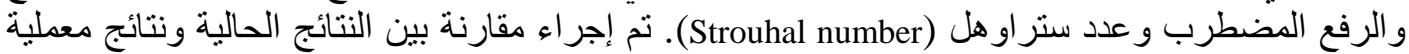

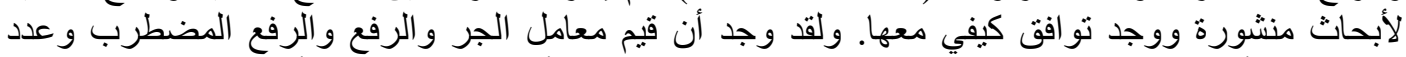

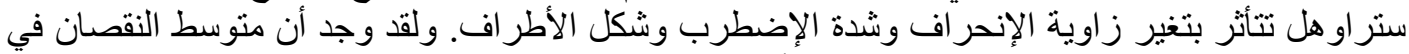

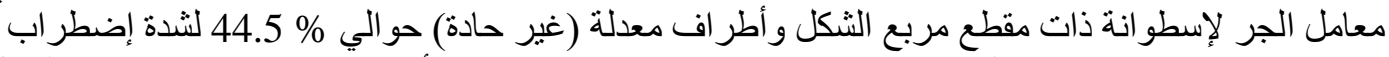

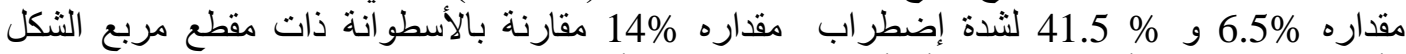

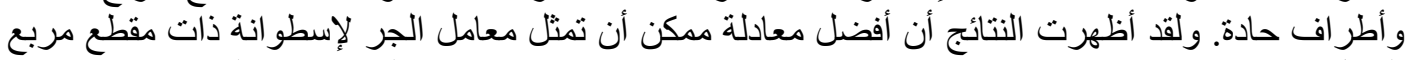

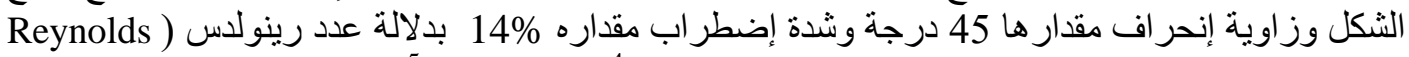

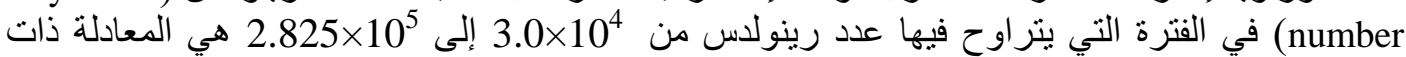

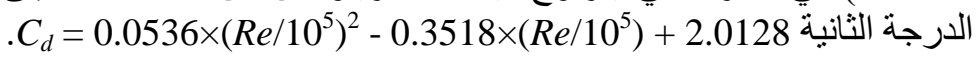

